\title{
Importância do uso de plantas medicinais nos processos de xerose, fissuras e cicatrização na diabetes mellitus
}

SILVA, L.L.'; LOPES, P.F.'; MONTEIRO, M.H.D.A.'; MACEDO, H.W."

Universidade Federal Fluminense, Rua Marquês do Paraná, 303, Departamento de Patologia, $4^{\circ}$ andar, CEP:24033-900, Niterói (RJ), Brasil. *Autor para correspondência: hwmacedo@terra.com.br

RESUMO: Artigo de revisão que trata da importância do tratamento da xerose e de fissuras nos pés de pacientes com diabetes mellitus. Essas fissuras se não tratadas, constituem porta de entrada para infecções e podem resultar na formação de úlceras, que são a causa mais comum das amputações de extremidades de causa não traumática. Por isso a prevenção de fissuras nos pés de diabéticos é fundamental, já que a cicatrização é um processo complicado nesses pacientes, devido à hiperglicemia. Aborda sobre produtos usados no tratamento de xerose e fissuras e o crescente interesse de pesquisadores e do mercado no uso de fitoterápicos com esse objetivo.

Palavras-chave: diabetes, fissuras, cicatrização de feridas, tratamento, plantas medicinais

\begin{abstract}
The importance of the use of medicinal plants in the process of xerosis, fissures and healing. A review article that deals with the importance of treating xerosis and fissures in the feet of patients with diabetes mellitus. These wounds can be a gateway to infections and may result in the formation of ulcers, if untreated. The ulcers are the most common cause for the amputations of extremities at non-traumatic cases. For this reason, the prevention of fissures in the feet of diabetic patientsis basal, since healing is a complicated process in this patients because of their hyperglycemia symptom. This article addresses the products employed in the therapy of xerosis and fissures, and also investigates the increasing interest of researchers and market in the application of phytotherapic medicines for this purpose.
\end{abstract}

Keywords: diabetes; fissures; wound healing; therapy; medicinal plants

\section{INTRODUÇÃO}

Mundialmente, um número crescente de indivíduos portadores de diabetes mellitus (DM) tem sido registrado e as complicações crônicas da doença, como por exemplo, o pé diabético, representam grande importância clínica. No Brasil a DM atinge em torno de $7,6 \%$ da população entre 30 e 69 anos de idade, sendo que $50 \%$ dos pacientes desconhecem o diagnóstico e $24 \%$ não fazem qualquer tipo de tratamento (Gross \& Nehme, 1999; Milman et al., 2001; Bortoletto et al., 2009).

A cada seis anos cresce em $18 \%$ o número de homens com DM e cerca de $20 \%$ dos idosos com mais de 65 anos têm a doença. Fatores de risco como a obesidade, o envelhecimento, mau controle glicêmico, tabagismo e hipertensão arterial sistêmica (HAS) contribuem para esse crescimento, chegando a mais de 87,9 milhões de reais ao ano, o custo das internações hospitalares com a doença na rede pública brasileira (SBACVRJ, 2013). As complicações comuns da DM são as lesões crônicas nos vasos sanguíneos (vasculopatias) e nos nervos (neuropatias) afetando principalmente rins, retina, artérias, cérebro e nervos periféricos (Gross \& Nehme, 1999; Bortoletto et al., 2009).

Uma das principais causas de hospitalização de pacientes com DM é a amputação de membros inferiores, um fator de incapacidade, invalidez, aposentadoria precoce e morte que implica em altos custos financeiros. Geralmente, a amputação ocorre em decorrência das alterações vasculares e/ou neurológicas peculiares da doença, que são responsáveis pelo "pé diabético" (Milman et al., 2001).

Pacientes com DM apresentam frequentemente xerose nos pés. Essa é uma desordem da pele caracterizada por ressecamento anormal, desconfortável e bastante frequente também em não diabéticos, como na dermatite atópica, na deficiência de vitaminas e em outras condições que levem ao ressecamento da pele, com alterações no equilíbrio da umidade e da oleosidade das camadas da derme (Barco \& Giménez-Arnau, 
2008) derme. A xerose afeta de $15 \%$ a $20 \%$ da população mundial, compromete a qualidade de vida do paciente e quando grave, pode interferir na produtividade no trabalho, especialmente quando as mãos são afetadas. Precisa ser tratada e a base do tratamento é a reidratação e a reparação da pele, muito importantes para recuperar a função de barreira epidérmica (Barco \& Gimenez, 2008; Onselen, 2011).

Os fatores desencadeantes além das doenças que causam ressecamento da pele são: idade, meio ambiente, exposições repetidas a solventes, sabões e desinfetantes, baixa umidade relativa do ar atmosférico e a exposição a ventos frios e secos. Essas situações levam à desidratação da camada córnea e à retirada dos lipídios da pele, destruindo assim a barreira cutânea e aumentando a perda de água. Contribuem também o uso de aquecedores de ambiente e a ventilação de cômodos com ar seco e frio (Onselen, 2011; Proksch \& Lachapelle, 2005).

Em pessoas idosas é uma condição comum, sendo que a predisposição genética, o uso de diuréticos e medicamentos contribuem, também, para o seu desenvolvimento. O exame físico revela uma pele áspera e seca ao toque, pode haver descamação e nos casos graves, vermelhidão e formação de fissuras e o prurido pode levar a escoriações e risco de infecções na pele. Os pacientes podem minimizar seu efeito aumentando a umidade do ambiente, modificando os produtos de banho e usando emolientes para substituir os componentes lipídicos da pele. São necessários cuidados para evitar a sensibilização da pele com produtos que provocam hipersenssibilidade, como lanolina e parabenos, comumente encontrados em cosméticos (White-Chu \& Reddy, 2011).

A xerose geralmente conduz ao desenvolvimento de fissuras, representando um fator de risco para o desenvolvimento de úlceras nos pés de pacientes diabéticos (Federici et al., 2012). Fissuras são pequenas fendas ou rachas na pele calosa das mãos ou dos pés causadas pela perda linear da epiderme e derme, em áreas de pregas ou dobras da pele, provenientes da eliminação ou destruição dos tecidos cutâneos, causadas basicamente pela perda da elasticidade da pele (Nunes et al., 2013). Fatores determinantes para o seu aparecimento são: ressecamento cutâneo, idade, hábitos alimentares, quantidade de água ingerida, uso de medicamentos, exposição da pele a agressões químicas e do solo, uso de meias e sapatos inadequados e a obesidade (Nunes et al., 2011).

Em pacientes diabéticos as fissuras nos pés são frequentes e o processo de cicatrização é lento e precário. Grande parte das complicações está relacionada a problemas nos vasos sanguíneos que, além de conterem a glicose, levam nutrientes e recolhem toxinas dos tecidos corporais. Esses vasos podem ser danificados pela alta glicemia, o que causa danos nos órgãos que irrigam e prejudicam o processo de reparo e cicatrização. Além disso, ocorre menor síntese de colágeno e lesão no tecido conjuntivo. (Mendonça et al., 2009).

Com a finalidade de evitar a formação de fissuras na pele de diabéticos, além de tratar o ressecamento da pele é necessária a manutenção de bons hábitos alimentares, controle da glicose sanguínea, cuidados e higiene pessoal, principalmente dos pés (uso de sapatos e meias apropriados; checagem diária da presença de ressecamento e fissuras (Caiafa et al., 2011). A anamnese é fundamental para verificar a presença de fatores de risco em pacientes considerados assintomáticos. Há a necessidade de avaliação dos membros inferiores, pois geralmente o paciente não valoriza dados importantes como calos, fissuras, micoses, etc. (Gross \& Nehme, 1999; Caiafa et al., 2011). Pacientes com história prévia de úlcera ou amputação têm elevado risco para o desenvolvimento de novas úlceras, infecção e gangrena, podendo culminar em amputação (Milman et al., 2001; Bortoletto et al., 2009; Marfella et al., 2012).

A cicatrização de feridas é um processo complexo que envolve a organização de células, sinais químicos e remodelamento da matriz extracelular, com o objetivo de reparar o tecido. $\mathrm{O}$ tratamento de feridas busca o fechamento rápido da lesão de forma a se obter cicatriz funcional e, esteticamente, satisfatória. Para tanto, é indispensável melhor compreensão do processo biológico envolvido na cicatrização de feridas e na regeneração tecidual (Mendonça et al., 2009).

Os elevados custos dos tratamentos de alterações relacionadas à deficiência cicatricial aumentam a importância das pesquisas em busca de medicamentos capazes de interagir com o tecido lesado, tendo por objetivo acelerar o processo. $\mathrm{O}$ retardo de cicatrização, como ocorre no caso de úlceras de pés diabéticos, constitui grave problema mundial, tanto financeiro quanto social (Mendonça \& Netto, 2009). As feridas nesses pacientes são lesões de difícil tratamento e os métodos atuais incluem o uso de antibióticos, enxertos de tecidos e enzimas proteolíticas, com grandes desvantagens e efeitos colaterais indesejados (Montes et al., 2009; Mendonça \& Netto, 2009).

Os produtos utilizados para a reparação cutânea devem possuir requisitos estruturais e funcionais peculiares, como não apresentar antigenotoxicidade; não provocar reação inflamatória; facilitar a colonização da parte das

Rev. Bras. PI. Med., Campinas, v.17, n.4, supl. I, p.827-835, 2015. 
células estromais e a síntese de glicoproteínas e favorecer a migração e a diferenciação das células epiteliais. Essas propriedades podem ser traduzidas como eficácia, segurança e versatilidade, que juntas propiciam também o conforto do paciente, a facilidade de aplicação ou remoção, a segurança médica e a relação positiva do custo/benefício com o uso clínico do produto (Paton et al., 2012).

Existem vários estudos que comprovam a eficácia de diferentes produtos no auxílio dos processos de reparo e cicatrização de lesões cutâneas, como o uso do ácido trans-retinóico que auxilia na produção de pró-colágeno tipo I. Embora os retinóides ofereçam ótimos resultados nesses processos, provocam grande irritação na pele da maioria dos indivíduos, gerando aumento do processo inflamatório e abandono do tratamento (Bhagavathula et al., 2009).

Papanas \& Maltezos (2011) publicaram um comentário sobre a importância das plantas como uma nova tentativa da medicina no combate às várias doenças e que os resultados têm sido promissores, principalmente na cicatrização de feridas, como as úlceras em diabéticos. Relataram que muitos hidratantes à base de plantas já foram estudados, sua eficácia comprovada na regeneração e na prevenção ao envelhecimento da pele.

Muitos estudos têm concentrado esforços no desenvolvimento de cosméticos usando como base plantas medicinais devido aos efeitos nocivos causados por muitos produtos químicos como o ácido trans-retinóico, exemplificado acima. Esses produtos têm sido usados no tratamento das feridas ao longo dos anos, por promoverem a coagulação do sangue, combaterem infecções e acelerarem o processo de cicatrização. As plantas têm um imenso potencial para o tratamento de feridas, sendo utilizadas há milênios por povos indígenas em muitos países. Os produtos a base de plantas são considerados mais econômicos, mais acessíveis e mais seguros (Thakur et al., 2011).

Formulações cosméticas poli-ervas veiculadas em cremes, géis e óleos vêm sendo recomendadas para tratamentos prolongados, e seus efeitos já são bem aceitos e comprovados para o tratamento de cicatrização de feridas na pele. Agem melhorando xerose, fissuras e promovendo a cicatrização da pele danificada (Bhagavathula et al., 2009; Reddy et al., 2012).

Uma vez que o estabelecimento da xerose antecede a formação de fissuras e feridas nos pés de pacientes com DM, seu tratamento é essencial como prevenção. $O$ tratamento da xerose é feito com uso repetido de hidratantes para manter o conteúdo de água da pele evitando a perda de elasticidade e o aparecimento de fissuras, muitas vezes dolorosas, que podem atuar como porta de entrada para infecções (Baalham et al., 2011). A instalação da infecção é uma condição ameaçadora ao membro e considerada uma causa imediata de amputação em 25 a $50 \%$ dos pacientes diabéticos (Federici et al., 2012; Seité et al., 2011).

Embora alguns produtos ocasionem alívio dos sintomas visíveis da secura na pele, a perda elevada e anormal de água transepidérmica tem sido relatada mesmo com o uso de certos hidratantes. Por isso, a escolha do produto correto para o tratamento é muito importante, sendo necessário o conhecimento sobre a sua composição e eficácia (Lodén, 2012). Os emolientes como a glicerina e o propilenoglicol são comumente usados para tratar a xerose em pacientes diabéticos. Quando usados corretamente são parte essencial do controle da dermatite e ajudam na cicatrização da pele (Seité et al., 2011). Existem também cremes à base de ureia, carnosina e arginina para o tratamento dessas lesões que proporcionam excelente hidratação da pele (Federici et al., 2012). No entanto, vários estudos demonstram a eficácia de diversos emolientes, cremes e hidratantes desenvolvidos à base de plantas medicinais, com essa finalidade sendo mais seguros e econômicos (Ahmad, 2010; Reddy et al., 2012).

Como a prevalência da xerose é alta no mundo, é necessário encontrar soluções novas e seguras para seu tratamento, uma vez que a hidratação diária da pele previne além do ressecamento, o desenvolvimento de fissuras. Como exemplos temos o óleo de canola (Brassica napus L.) e a Aloe vera L. (Lodén, 2012) além do óleo de girassol (Helianthus annuus L.), de copaíba (Copaifera langsdorffii), barbatimão (Stryphnodendron adstringens) e aroeira (Schinus molle L.), sendo que o curativo feito com hidrogel é uma opção de tratamento para ajudar a fechar úlceras no pé diabético (Dumville et al., 2013). Todos esses vegetais podem ser usados em substituição à colagenase ou outro medicamento similar, na recuperação cutânea.

No Centro de Pesquisa da Diabetes de Chennai, entre 2008 e 2009, foi desenvolvido um estudo com pacientes diabéticos tipo 2 com úlceras nos pés desde superficiais até profundas. Para o tratamento das lesões foi avaliada a eficácia de um novo creme natural composto por uma mistura de várias ervas como Aloe vera L., Pandanus odoratissimus L., Curcuma longa L., Cocos nucifera L., Glycyrrhiza glabra L., Musa paradisíaca L.. Como controle usaram creme de sulfadiazina de prata, um bactericida derivado das sulfamidas muito utilizado para tratar úlceras em diabéticos. O novo creme mostrou-se mais eficaz na redução da ferida, na síntese de colágeno, além da ação microbicida e antiinflamatória, representando um tratamento

Rev. Bras. PI. Med., Campinas, v.17, n.4, supl. I, p.827-835, 2015. 
alternativo para úlceras (Viswanathan et al., 2011).

A busca por terapias alternativas para promover a cicatrização de feridas tem sido intensificada mesmo em países desenvolvidos, enquanto as modernas terapias com antibióticos e corticóides têm sido preteridas devido aos efeitos colaterais que medicamentos alopáticos podem acarretar. Aproximadamente $60 \%$ da população mundial utiliza quase que inteiramente plantas para a medicação e os produtos naturais têm sido reconhecidos como uma fonte importante de medicamentos terapeuticamente eficazes (Kumar et al., 2007). Como exemplo temos Jatropha curcas L. (Euphorbiaceae), Aloe barbadensis Mill. (Xanthorrhoeaceae), e Centella asiatica L. (Apiaceae), que exibem além da propriedade cicatrizante, outras como atividade antimicrobiana, antioxidante, antifúngica e antiinflamatória (Hayouni et al., 2011).

Baseado nessa busca por terapias alternativas para o tratamento de xerose, fissuras e cicatrização de feridas no pé diabético, o objetivo desse estudo foi identificar na literatura existente sobre o tema, algumas das principais plantas medicinais que vem sendo estudadas com esse objetivo.

\section{MÉTODO}

Foi realizado um levantamento sobre o tema nas principais fontes de pesquisa científica no período de 2006 a 2014, usando os descritores consultados no DECs (Descritores em Ciência da Saúde): diabetes; fissures; wound healing; therapy; medicinal plants e seus correspondentes em português, diabetes; fissuras; cicatrização de feridas; tratamento; plantas medicinais (Tabela 1).

Os artigos relacionados com o tema que foram selecionados, apresentavam e dicutiam sobre a importância do uso de plantas medicinais no tratamento de xerose, fissuras e feridas na pele, alterações importantes em pacientes com DM. Aqueles artigos que não se encaixavam exatamente no tema dessa revisão foram excluidos após leitura do resumo.

\section{RESULTADOS E DISCUSSÃO}

Nas Tabelas 2 e 3 estão listados os artigos relacionados com o tema (utilização de plantas medicinais nos processos de xerose e cicatrização de lesões cutâneas) em diferentes partes do mundo e no Brasil, respectivamente.

De um total de 45 artigos foram encontrados apenas dois com objetivo de estudar a xerose. Oleum amygdalae e Arctium lappa L. foram considerados importantes para tratar xerose em humanos (Ahmad, 2010; Lee et al., 2013). Esse reduzido número de publicações sobre o tema mostra a necessidade de novos estudos sobre plantas medicinais importantes para o tratamento dessa alteração que ocorre com frequência na pele de pacientes com DM.

Apenas seis trabalhos dos artigos listados nas tabelas 2 e 3 foram desenvolvidos em seres humanos, possivelmente devido às dificuldades na aprovação de execução dos projetos pelos Comitês de Ética em Pequisa com seres humanos, ou ainda, pela dificuldade inerente de controle sobre o seguimento do tratamento por esses indivíduos. Um dos artigos mostrou que o óleo de raiz de Arnebia tem efeito positivo na promoção do fator de crescimento de fibroblasto, que pode regular o processo de reparo tecidual (Pei et al., 2007). Para o tratamento da xerose Oleum amydalae e Actium lappa L. são indicados (Ahmad, 2012; Lee et al., 2013) enquanto que Aloe vera mostrou-se eficaz para cicatrização de feridas (Eshghi et al., 2010; Oliveira et al., 2010; Pereira et al., 2014).

O modelo experimental animal (rato ou camundongo) foi utilizado pelos 39 artigos restantes, todos empregando plantas medicinais em processo de cicatrização de feridas. Cada um deles foi desenvolvido com uma determinada espécie vegetal, excetuando Aloe vera e Punica granatun L. que foram estudadas por mais de um grupo de pesquisadores, usando principalmente modelo de cicatrização de feridas cutâneas em ratos (Tabelas 2 e 3). Observa-se, portanto, a necessidade de novos estudos sobre a comprovação da eficácia de cada uma dessas plantas medicinais na cicatrização de feridas na pele, principalmente em modelos humanos.

Países como Índia e China conhecidos pela prática da medicina não tradicional, lideram

TABELA 1. Resultado do levantamento realizado sobre o tema nas principais fontes de pesquisa

\begin{tabular}{ccccc}
\hline Base de dados & Total de artigos & $\begin{array}{c}\text { Exclusão após leitura } \\
\text { do resumo }\end{array}$ & Lidos na íntegra & Selecionados \\
\hline PUBMED & 80 & 20 & 60 & 60 \\
THIEME & 3 & 3 & 0 & 0 \\
EBSCO Host & 4 & 2 & 2 & 2 \\
SCIELO & 28 & 15 & 13 & 13 \\
\hline \hline Total & 115 & 40 & 75 & 75 \\
\hline
\end{tabular}

Rev. Bras. PI. Med., Campinas, v.17, n.4, supl. I, p.827-835, 2015. 
TABELA 2. Levantamento de estudos clínicos e experimentais sobre a utilização de plantas medicinais nos processos de xerose e cicatrização de lesões cutâneas em diferentes regiões do mundo

\begin{tabular}{|c|c|c|c|c|c|c|c|c|}
\hline Autores & Ano & Local & Espécime & Planta(s) & $\begin{array}{l}\text { Parte } \\
\text { usada }\end{array}$ & Aplicação & $\begin{array}{l}\text { Forma } \\
\text { usada }\end{array}$ & $\begin{array}{l}\text { Constituintes da } \\
\text { planta }\end{array}$ \\
\hline Gupta et al. & 2006 & Índia & rato & Rhodiola imbricata & rizoma & cicatrização de ferida & extrato & Polifenois \\
\hline Nayak e Pinto Pereira & 2006 & Trinidad & rato & Catharanthus roseus & flor & cicatrização de ferida & extrato & alcaloides e taninos \\
\hline Nakayak et al & 2007 & Trinidad & rato & Matricaria & flor & cicatrização de ferida & extrato & polifenois e flavonoides \\
\hline Pei et al. & 2007 & China & humano & Arnebia root & raiz & cicatrização de ferida & óleo & Alcaloides \\
\hline Abdulla et al. & 2009 & Malásia & rato & Rafflesia hassltii & $\begin{array}{l}\text { botão e } \\
\text { flor }\end{array}$ & cicatrização de ferida & extrato & Taninos \\
\hline Bhagavathula et al. & 2009 & EUA & rato & $\begin{array}{l}\text { Curcumin longa } \\
\text { Zingiber officinale }\end{array}$ & raiz & cicatrização de ferida & solução & curcumenoides e gingerois \\
\hline Nayak et al. & 2009 & Trinidad & rato & Morinda citrifolia L & folha & cicatrização de ferida & extrato & triterpenoides e alcaloides \\
\hline Sheeaba et al & 2009 & Índia & rato & $\begin{array}{l}\text { Cassia occidentalis } \\
\text { L. }\end{array}$ & folha & cicatrização de ferida & extrato & Alcaloides \\
\hline Ahmad & 2010 & $\begin{array}{l}\text { Reino } \\
\text { Unido }\end{array}$ & humano & Oleum amygdalae & amêndoa & xerose & óleo & $\begin{array}{l}\text { beta-zoosterol, esqualeno, } \\
\text { alfa-tocoferol }\end{array}$ \\
\hline Eshghi et al & 2010 & Irã & humano & Aloe vera & folha & cicatrização de ferida & creme & Polissacarídeos \\
\hline Pirbalouti & 2010 & Irã & rato & $\begin{array}{l}\text { Malva sylvestris; } \\
\text { Punica granatum }\end{array}$ & flor & cicatrização de ferida & extrato & Polifenois \\
\hline Hayoni et al. & 2011 & Tunísia & cobaia & Punica granatum L. & casca & cicatrização de ferida & extrato & tanino, polifenois \\
\hline Lai et al. & 2011 & Malásia & rato & $\begin{array}{l}\text { Blechnum orientale } \\
\text { Linn }\end{array}$ & folha & cicatrização de ferida & extrato & Taninos \\
\hline Nayak et al. & 2011 & Trinidad & rato & Carapa guianensis & folha & cicatrização de ferida & extrato & $\begin{array}{l}\text { taninos, saponinas, } \\
\text { alcaloides }\end{array}$ \\
\hline Bhaskar \& Nithya & 2012 & Índia & rato & $\begin{array}{l}\text { Hibiscus rosa } \\
\text { sinensis } L \text {. }\end{array}$ & flor & cicatrização de ferida & extrato & $\begin{array}{l}\text { flavonoides, saponinas, } \\
\text { terpenoides e taninos }\end{array}$ \\
\hline Chen et al. & 2012 & China & rato & Lonicera japonica & flor & cicatrização de ferida & Extrato & $\begin{array}{l}\text { flavonoides, saponinas, } \\
\text { iridoides }\end{array}$ \\
\hline Dewangan et al. & 2012 & Índia & rato & $\begin{array}{l}\text { Solanum } \\
\text { xanthocarpum } \\
\text { schrad and wendl }\end{array}$ & folha & cicatrização de ferida & extrato & $\begin{array}{l}\text { alcaloides, sapononas, } \\
\text { glicosideos }\end{array}$ \\
\hline Fikru et al. & 2012 & Etiópia & rato & $\begin{array}{l}\text { Achyranthes aspera } \\
\text { L. }\end{array}$ & folha & cicatrização de ferida & extrato & Saponinas \\
\hline Ghosh et al. & 2012 & Índia & rato & $\begin{array}{l}\text { Pedilanthus } \\
\text { tithymaloides L. }\end{array}$ & folha & cicatrização de ferida & extrato & $\begin{array}{l}\text { 2-(3,4-dihydroxy-phenyl)-5; } \\
\text { 7-dihydroxy-chromen-4- } \\
\text { one; 1,2-tetradecanediol; } \\
\text { 1-(hydrogen sulfate); sodium } \\
\text { salt }\end{array}$ \\
\hline Natanzi et al. & 2012 & Irã & rato & $\begin{array}{l}\text { Elaeagnus } \\
\text { angustifolia }\end{array}$ & fruto & cicatrização de ferida & extrato & $\begin{array}{l}\text { flavonoides, terpinoides, } \\
\text { glicosideos }\end{array}$ \\
\hline Suntar et al. & 2012 & Turquia & rato & Pinus species & $\begin{array}{l}\text { pinha e } \\
\text { folha }\end{array}$ & cicatrização de ferida & óleo & monoterpenos \\
\hline Agyre et al. & 2013 & Africa & rato & Kigelia africana & $\begin{array}{l}\text { folha e } \\
\text { caule }\end{array}$ & cicatrização de ferida & extrato & $\begin{array}{l}\text { alcaloides, saponinas, } \\
\text { taninos, flavonoides }\end{array}$ \\
\hline Greethalakshmi et al. & 2013 & índia & rato & $\begin{array}{l}\text { Sphaeranthus } \\
\text { amarsnthoides }\end{array}$ & planta total & cicatrização de ferida & extrato & $\begin{array}{l}\text { taninos, flavonoides, } \\
\text { glicosideos, triterpenoides }\end{array}$ \\
\hline Khan et al. & 2013 & índia & rato & Aloe vera & folha & cicatrização de ferida & extrato & polisacarídeos \\
\hline Lee et al. & 2013 & Coreia & humano & Arctium lappa L. & semente & xerose & extrato & polifenois \\
\hline Murthy et al. & 2013 & índia & rato & Bacopa monniera & planta total & cicatrização de ferida & extrato & $\begin{array}{l}\text { saponinas, glicosideos e } \\
\text { alcaloides }\end{array}$ \\
\hline Qu et al. & 2013 & China & rato & Amorpha fruticosa $\mathrm{L}$. & fruto & cicatrização de ferida & $\begin{array}{l}\text { fruto } \\
\text { fresco }\end{array}$ & rotenoides \\
\hline Nayak et al. & 2014 & Trinidad & rato & Neurolaena lobata L. & folha & cicatrização de ferida & Extrato & $\begin{array}{l}\text { alcaloides e flavonoides, } \\
\text { saponinas, taninos }\end{array}$ \\
\hline
\end{tabular}

junto com o Irã e Trinidad o número de publicações sobre o uso de plantas medicinais no tratamento e cicatrização de xerose e feridas na pele (Tabela 2). No Brasil, o maior detentor de reserva vegetal do mundo, temos nos últimos cinco anos, 17 artigos publicados com relevância sobre o assunto (Tabela $3)$.

Em trabalho recente foi relatado que o Ministério da Saúde brasileiro vem buscando estímular à inserção de plantas medicinais no sistema de saúde, através de políticas nacionais (Piriz et al, 2014). Como há no mundo um número crescente de indivíduos com DM e suas complicações, pesquisas sobre plantas medicinais eficazes no tratamento das lesões do pé diabético devem ser estimuladas (Gross \& Nehme, 1999).

O potencial medicinal de uma espécie deve-se à presença de princípios ativos capazes de

Rev. Bras. PI. Med., Campinas, v.17, n.4, supl. I, p.827-835, 2015. 
produzir efeitos farmacológicos diversos, tais como analgésicos, antissépticos, diuréticos, calmantes, cicatrizantes, emolientes, entre outros. Dentre as substâncias bioativas que podem ser encontradas nas diversas partes de uma planta, podem ser citadas: alcalóides, saponinas, taninos, glicosídeos, flavonóides e óleos essenciais (Santos \& Torres, 2012). Essas substâncias foram encontradas nas plantas medicinais estudadas na literatura pesquisada (Tabelas 2 e 3 ).

O Brasil possui a maior reserva florestal diversificada do planeta. Muitas destas espécies utilizadas para fins medicinais são empregadas com pouca ou nenhuma comprovação de suas propriedades farmacológicas. O uso de plantas medicinais não se restringe apenas às zonas rurais ou regiões desprovidas de assistência médica e farmacêutica. São também utilizadas intensamente no meio urbano, como forma alternativa ou complementar aos medicamentos alopáticos
(Santos et al., 2012). O interesse na descoberta de novas substâncias faz com que cientistas de várias áreas busquem, na flora brasileira, espécies vegetais com propriedades medicinais utilizadas pela população (Santos \& Torres, 2012).

Observamos, portanto, que existe um interesse mundial no estudo sobre a utilização de diferentes plantas medicinais de diferentes regiões, para o tratamento de alterações na pele como xerose e feridas na pele causadas por diferentes processos e, portanto, no tratamento de lesões do pé diabético.

\section{CONCLUSÕES}

Após esse estudo podemos concluir que pacientes com DM tem tendência a apresentar xerose nos pés, contribuindo para o desenvolvimento de fissuras e formação posterior de úlceras e que existem vários produtos à base de plantas com

TABELA 3. Levantamento de estudos clínicos e experimentais sobre a utilização de plantas medicinais nos processos de xerose e cicatrização de lesões cutâneas no Brasil

\begin{tabular}{|c|c|c|c|c|c|c|c|c|}
\hline Autores & Ano & U.F. & Espécime & Planta(s) & Parte usada & Aplicação & $\begin{array}{l}\text { Forma } \\
\text { usada }\end{array}$ & Constituinte da planta \\
\hline $\begin{array}{l}\text { Mendonça } \\
\text { et al. }\end{array}$ & 2009 & SP & rato & Aloe vera $\mathrm{L}$. & Folhas & $\begin{array}{l}\text { cicatrização de feridas } \\
\text { cutâneas }\end{array}$ & gel & $\begin{array}{l}\text { antraquinonas, } \\
\text { polissacarídeos }\end{array}$ \\
\hline Oliveira et al. & 2010 & CE & humano & Aloe vera $\mathrm{L}$. & Folhas & $\begin{array}{l}\text { cicatrização de feridas } \\
\text { cutâneas }\end{array}$ & extrato & fenois, polissacarídeos \\
\hline Duarte et al. & 2011 & SP & rato & Chamomilla recutita L. & Flores & $\begin{array}{l}\text { cicatrização de feridas } \\
\text { cutâneas }\end{array}$ & extrato & fenois, flavonoides \\
\hline Franco et al. & 2011 & PE & rato & Linum usitatissimum L. & Semente & $\begin{array}{l}\text { cicatrização de feridas } \\
\text { cutâneas }\end{array}$ & óleo & $\begin{array}{l}\text { ácidos glaxos } \\
\text { polissaturados, acidos } \\
\text { glaxos essenciais }\end{array}$ \\
\hline $\begin{array}{l}\text { Cavalcanti } \\
\text { et al. }\end{array}$ & 2012 & CE & camundongo & Croton zehntneri & Folhas & $\begin{array}{l}\text { cicatrização de feridas } \\
\text { cutâneas }\end{array}$ & óleo & trans-anethole \\
\hline Cesca et al. & 2012 & SC & camundongo & Aleurites moluccana L. & Folhas & $\begin{array}{l}\text { cicatrização de feridas } \\
\text { cutâneas }\end{array}$ & extrato & Flavonoides \\
\hline $\begin{array}{l}\text { Trivellato } \\
\text { Grassi et al. }\end{array}$ & 2013 & SC & camundongo & $\begin{array}{l}\text { Chenopodium } \\
\text { ambrosioides }\end{array}$ & folhas, caule & $\begin{array}{l}\text { cicatrização de feridas } \\
\text { cutâneas }\end{array}$ & extrato & terpenos, esteróides,fenóis \\
\hline Pessoa et al. & 2012 & PE & rato & $\begin{array}{l}\text { Anadenanthera colubrina } \\
\text { var. cebil }\end{array}$ & $\begin{array}{l}\text { casca, } \\
\text { entrecasca }\end{array}$ & $\begin{array}{l}\text { cicatrização de feridas } \\
\text { cutâneas }\end{array}$ & extrato & taninos \\
\hline $\begin{array}{l}\text { Passarini } \\
\text { et al. }\end{array}$ & 2012 & SP & rato & Jatropha curcas L. & Semente & $\begin{array}{l}\text { cicatrização de feridas } \\
\text { cutâneas }\end{array}$ & óleo & $\begin{array}{l}\text { tanino, flavonóides, } \\
\text { isoflavonóides, e outros } \\
\text { compostos fenólicos }\end{array}$ \\
\hline Batista et al. & 2012 & RN & rato & Mauritia flexuosa L. & Fruto & $\begin{array}{l}\text { cicatrização de feridas } \\
\text { cutâneas }\end{array}$ & óleo & $\begin{array}{l}\text { carotenódes, ácidos graxos, } \\
\text { tocoferol }\end{array}$ \\
\hline Coelho et al. & 2013 & SP & rato & $\begin{array}{l}\text { Cenostigma } \\
\text { macrophyllum Tul. }\end{array}$ & Semente & $\begin{array}{l}\text { cicatrização de feridas } \\
\text { cutâneas }\end{array}$ & $\begin{array}{l}\text { emulsão } \\
\text { óleo- } \\
\text { água }\end{array}$ & $\begin{array}{l}\text { flavonóides, isoflavonóides, } \\
\text { rotenoides }\end{array}$ \\
\hline Sauaia et al. & 2013 & MA & camundongo & Euphorbia tirucalli L. & Látex & $\begin{array}{l}\text { cicatrização de feridas } \\
\text { cutâneas }\end{array}$ & extrato & triterpenos, fitosterol \\
\hline Agra et al. & 2013 & $A L$ & camundongo & Bowdichia virgilioides & caule, casca & $\begin{array}{l}\text { cicatrização de feridas } \\
\text { cutâneas }\end{array}$ & extrato & benzofurano, alcaloides \\
\hline $\begin{array}{l}\text { Estevão } \\
\text { et al. }\end{array}$ & 2013 & PEI & rato & $\begin{array}{l}\text { Schinus terebinthifoliu } \\
\text { Raddi }\end{array}$ & Folha & $\begin{array}{l}\text { cicatrização de feridas } \\
\text { cutâneas }\end{array}$ & óleo & verbenona, carvona \\
\hline Rebolla et al. & 2013 & SP & rato & Brassica oleracea & Folha & $\begin{array}{l}\text { cicatrização de feridas } \\
\text { cutâneas }\end{array}$ & extrato & $\begin{array}{l}\text { fenois, flavonóides, } \\
\text { triterpenos e esteroides }\end{array}$ \\
\hline Aro & 2013 & SP & rato & Arrabidaea chica & Folhas & $\begin{array}{l}\text { cicatrização de feridas } \\
\text { cutâneas }\end{array}$ & extrato & antocianinas \\
\hline Pereira et al. & 2014 & RS & humano & Aloe vera $\mathrm{L}$. & Folha & $\begin{array}{l}\text { cicatrização de feridas } \\
\text { cutâneas }\end{array}$ & extrato & $\begin{array}{l}\text { antraquinonas, fenois, } \\
\text { polissacarídeos }\end{array}$ \\
\hline
\end{tabular}

U.F.: unidade da Federação

Rev. Bras. PI. Med., Campinas, v.17, n.4, supl. I, p.827-835, 2015. 
propriedades pró-cicatrizantes e curativas, que vem sendo usados para o tratamento de lesões na pele. Tais fitoterápicos melhoram a coagulação sanguínea, combatem a infecção e aceleram a cicatrização e, vários óleos essenciais extraídos de plantas apresentam também, propriedades prócicatrizantes e curativas, e vêm, portanto, sendo utilizados em fármacos para o tratamento de xerose e fissuras, com comprovada eficácia e redução dos efeitos secundários causados por muitos produtos químicos.

\section{REFERÊNCIAS}

ABDULLA, M.A. et al. Wound healing activities of Rafflesia hasseltii extract in rats. Journal of Clinical Biochemistry and Nutrition, v.45, n.3, p.304-308, 2009.

AGRA, I.K. et al. Evaluation of wound healing and antimicrobial properties of aqueous extract from Bowdichia virgilioides stem barks in mice. Anais da Academia Brasileira de Ciênciais, v.85, n.3, p.945954, 2013.

AGYRE, C. et al. Antimicrobial, Antioxidant, and Wound Healing Properties of Kigelia africana (Lam.) Beneth. and Strophanthus hispidus DC. Advances in Pharmacological Sciences, v.2013, n.2013, p.1-10, 2013.

AHMAD, Z. The uses and properties of almond oil. Complementary Therapies in Clinical Practice, v.16, n.1, p.10-12, 2010.

ARO, A.A. et al. Effect of the Arrabidaea chica extract on collagen fiber organization during healing of partially transected tendo. Life Sciences, v.92, n.13, p.799807, 2013

BAALHAM, P. et al. Xerosis of the feet: a comparative study on the effectiveness of two moisturizers. British J ournal of Community Nursing, v.16, n.12, p.591597, 2011.

BARCO, D.; GIMÉNEZ-ARNAU, A. Xerosis: a dysfunction of the epidermal barrier. Actas Dermo-Sifiliográficas, v.99, n.9, p.671-682, 2008.

BATISTA, J.S. et al. Atividade antibacteriana e cicatrizante do óleo de buriti Mauritia flexuosa L. Ciência Rural, v.42, n.1, p.136-141, 2012.

BHAGAVATHULA, $\mathrm{N}$. et al. A combination of curcumin and ginger extract improves abrasion wound healing in corticosteroid-damaged hairless skin. Wound Repair and Regeneration, v.17, n.3, p.360-366, 2009.

BHASKAR, A.; NITHYA, V. Evaluation of the woundhealing activity of Hibiscus rosa sinensis $L$ (Malvaceae) in Wistar albino rats. Indian Journal of Pharmacology, v.44, n.6, p.694-698, 2012.

BORTOLETTO, M.S.S. et al. Pé diabético, uma avaliação sistematizada. Arquivo de Ciências da Saúde Unipar, v.13, n.1, p.37-43, 2009.

CAIAFA, J.S. et al. Atenção integral ao portador de pé diabético. Jornal Vascular Brasileiro, v.10, n.4, p.132, 2011.

CAVALCANTI, J.M. et al. The essential oil of Croton zehntneri and trans-anethole improves cutaneous wound healing. Journal of Ethnopharmacology, v.144, n.2, p.240-247, 2012.

CESCA, T.G. et al. Antinociceptive, anti-inflammatory and wound healing features in animal models treated with a semisolid herbal medicine based on Aleurites moluccana L. Willd. Euforbiaceae standardized leaf extract: semisolid herbal. Journal Ethnopharmacology, v.143, n.1, p.355-362, 2012.

CHEN, W.C. et al. Wound repair and anti-inflammatory potential of Lonicera japonica in excision wound-induced rats. BMC Complementary and Alternative Medicine, v.12, n.226, p.1-9, 2012.

COELHO, N.P. et al. Cenostigma macrophyllum Tul. on the healing of skin wounds in rats with diabetes mellitus. Acta Cirurgica Brasileira, v.28, n.8, p.594-600, 2013.

DEWANGAN, $\mathrm{H}$. et al. Potential wound healing activity of the ethanolic extract of Solanum Xanthocarpum schrad and wendl leaves. Pakistan Journal of Pharmaceutical Sciences, v.25, n.1, p.189-194, 2012.

DUARTE, C.M. et al. Effects of Chamomilla recutita (L.) on oral wound healing in rats. Medicina Oral Patologia Oral y Cirugia Bucal, v.16, n.6, p.716-21, 2011.

DUMVILLE, J.C. et al. Hydrogel dressings for healing diabetic foot ulcers. Cochrane Database Of Systematic Reviews, v.7, n.9, p.1-54, 2013.

ESHGHI, F. et al. Effects of Aloe vera cream on posthemorrhoidectomy pain and wound healing: results of a randomized, blind, placebo-control study. The Jornal of Alternative Complementary Medicine, v.16, n.6, p.647-650, 2010.

ESTEVÃO, L.R.M. et al. Effects of aroeira (Schinus terebinthifoliu Raddi) oil on cutaneous wound healing in rats. Acta Cirurgica Brasileira, v.28 n.3, p.1-8, 2013.

FEDERICI, A. et al. An urea and carnosine based cream (Ureadin Rx Db ISDIN) shows greater efficacy in the treatment of severe xerosis of the feet in type 2 diabetic patients in comparison with glycerol-based emollient cream. BioMed Central Dermatology, v.12, n.16, p.1-6, 2012.

FIKRU, A. et al. Evaluation of in vivo wound healing activity of methanol extract of Achyranthes aspera L. Jornal Ethnopharmacology, v.143, n.2, p.469-474, 2012.

FRANCO, E.S. et al. Effect of a Semisolid Formulation of Linum usitatissimum L. (Linseed) Oil on the Repair of Skin Wounds. Evidence-Based Complementary and Alternative Medicine, v.2012, n.2012, p.1-7, 2012.

GEETHALAKSHMI, R. et al. R. Evaluation of Antioxidant and Wound Healing Potentials of Sphaeranthus amaranthoides Burm.f. BioMed Research International, v.2013, n.2013, p.1-7, 2013.

$\mathrm{GHOSH}, \mathrm{S}$. et al. Evaluation of the wound healing activity of methanol extract of Pedilanthus tithymaloides (L.) poit leaf and its isolated active constituents in topical formulation. Jornal Ethnopharmacology, v.142, n.3, p.714-722, 2012.

GROSS, J.L.; NEHME, M. Detecção e tratamento das complicações crônicas do diabetes melito: consenso da Sociedade Brasileira de diabetes e Conselho Brasileiro de Oftalmologia. Revista da Associação Médica Brasileira, v.45, n.3, p.279-284, 1999

GUPTA, A. et al. Poly-herbal formulation accelerates normal and impaired diabetic wound healing. Wound Repair Regeneration, v.16, n.6, p.784-790, 2008. 
HAMMAM, J.H. Composition and Applications of Aloe vera Leaf Gel. Molecules, v.13 n.8, p.1599-1616, 2008.

HAYOUNI, E.A. et al. Hydroalcoholic extract basedointment from Punica granatum L. peels with enhanced in vivo healing potential on dermal wounds. International Journal of Phytotherapy and Phytopharmacology, v.18, n.11, p.976-984, 2011.

KHAN, A.W. et al. Formulation development, optimization and evaluation of aloe vera gel for wound healing. Pharmacognosy Magazine, v.9, n.36, p.6-10, 2013.

KUMAR, B. et al. Ethnopharmacological approaches to wound healing-exploring medicinal plants of India. Journal of Ethnopharmacology, v.114, n.2, p.103113, 2007.

LAI, H.Y. et al. Potential dermal wound healing agent in Blechnum orientale Linn. BMC Complementary and Alternative Medicine, v.11, n.62, p.1-5, 2011.

LEE, D.H. et al. The efficacy and safety of a proposed herbal moisturising cream for dry skin and itch relief: a randomised, double-blind, placebo-controlled trialstudy protocol. BMC Complementary and Alternative Medicine, v.13, n.330, p.1-7, 2013.

LODÉN, M. Effect of moisturizers on epidermal barrier function. Clinics in Dermatology, v.30, n.3, p.286296, 2012.

MARFELLA, R. et al. Dipeptidyl peptidase 4 inhibition may facilitate healing of chronic foot in patients with type 2 diabetes. Experimental Diabetes Research, v.2012, n.2012, p.1-11, 2012.

MENDONÇA, F.A.S. et al. Effects of the application of Aloe vera (L.) and microcurrent on the healing of wounds surgically induced in Wistar rats. Acta Cirurgica Brasileira, v.24, n.2, p.150-155, 2009.

MENDONÇA, R.J.; NETTO, J.C. Aspectos celulares da cicatrização. Anais Brasileiros em Dermatologia, v.84, n.3, p.257-262, 2009.

MIKAILI, P. et al. Pharmacological properties of herbal oil extracts used in Iranian traditional medicine. Advances in Environmental Biology, v.6, n.1, p.153-158, 2012.

MILMAN, M.H.S.A. et al. Pé diabético: avaliação da evolução e custo hospitalar de pacientes internados no conjunto hospitalar de Sorocaba. Arquivos Brasileiros de Endocrinologia e Metabologia, v.45, n.5, p.447451, 2001.

MONTES, L.V. et al. Evidências para o uso da óleo-resina de copaíba na cicatrização de ferida - uma revisão sistemática. Natureza on Line, v.7, n.2, p.61-67, 2009.

MURTHY, S. et al. Evaluation of In Vivo Wound Healing Activity of Bacopa monniera on Different Wound Model in Rats. BioMed Research International, v.2013, n.2013, p.1-9, 2013.

NATANZI, M.M. et al. Effect of aqueous extract of Elaeagnus angustifolia fruit on experimental cutaneous wound healing in rats. Acta Medica Iranica, v.50, n.9, p.589-596, 2012.

NAYAK, B.S.; PINTO PEREIRA, L.M. Catharanthus roseus flower extract has wound-healing activity in Sprague Dawley rats. BMC Complementary and Alternative Medicine, v. 6, n.41, p.1-6, 2006.

NAYAK, B.S. et al. Wound healing activity of Matricaria recutita L. extract. Jornal of Wound Care, v.16, n.7, p.298-302, 2007.

NAYAK, B.S. et al. Evaluation of the Wound-healing
Activity of Ethanolic Extract of Morinda citrifolia L. Leaf. Evidence-Based Complementary and Alternative Medicine, v.6, n.3, p.351-356, 2009.

NAYAK, B.S. et al. Experimental evaluation of ethanolic extract of Carapa guianensis L. leaf for its wound models. Evidence-Based Complementary and Alternative Medicine, v.2011, n.2011, p.1-6, 2011.

NAYAK, B.S. et al. Neurolaena lobata L. promotes wound healing in Sprague Dawley rats. International Journal of Applied and Basic Medical Research, v.4, n.2, p.106-110, 2014.

NUNES, J.C. et al. As principais causas de fissuras nos pés, 2011. Disponível em: http://Siaibib01.univali. $\mathrm{br} / \mathrm{pdf} / J$ essica Nunes, Jessica Marcelino.pdf. Acesso em: 21 jul 2013.

OLIVEIRA, S.H.S. et al. Use of collagen and Aloe vera in ischemic wound treatment: study case. Revista da Escola de Enfermagem da USP, v.44, n.2, p.344-349, 2010.

ONSELEN, J.V. Dry skin condition an evidenced-based focus on natural oatmeal emollients. Primary Health Care, v.21, n.2, p.31-38, 2011.

PAPANAS, N.; MALTEZOS, E. Polyherbal formulation as a therapeutic option to improve wound healing in the diabetic foot. The Indian Journal of Medical Research, v.134, n.2, p.146-147, 2011.

PASCALE, R. et al. Aggiornamenti in tema di infezioni del piede diabético. Infezioni in Medicina, n.3, p.155-168, 2012.

PASSARINI, J.R. et al. Application of Jatropha curcas L. seed oil (Euphorbiaceae) and microcurrent on the healing of experimental wounds in Wistar rats. Acta Cirurgica Brasileira, v.27, n.7, p.441-447, 2012.

PATON, J.S. et al. A comparison of customized and prefabricated insoles to reduce risk factors for neuropathic diabetic foot ulceration: a participant-blinded randomized controlled trial. Journal of Foot and Ankle Research, v.5, n.1, p.1-11, 2012.

PEI, X.W.et al. Clinical experimental study of Arnebia Root oil in increasing FGF expression and promoting wound surface healing. Journal of Nanjing Medical University, v.21, n.5, p.317-320, 2007.

PEREIRA, G.G. et al. Polymeric Films Loaded with Vitamin $E$ and Aloe vera for Topical Application in the Treatment of Burn Wounds. BioMed Research International, v.2014, n.2014, p.1-9, 2014.

PESSOA, W.S. et al. Effects of angico extract (Anadenanthera colubrina var. cebil) in cutaneous wound healing in rats. Acta Cirúrgica Brasileira, v.27, n.10, p.655-670, 2012.

PIRBALOUTI, A.G. et al. Wound healing activity of Malva sylvestris and Punica granatum in alloxan-induced diabetic rats. Acta Poloniae Pharmaceutica, v.67, n.5, p.511-516, 210.

PIRIZ, M. A.; LIMA, C.A.B. Plantas medicinais no processo de cicatrização de feridas: uma revisão de literature. Revista Brasileira de Plantas Medicinais, v.16, n.3, p.628-636, 2014.

PROKSCH, E.; LACHAPELLE, J.M. The management of dry skin with topical emollients-recent perspectives. Journal Der Deutschen Dermatologischen Gesellschaft, v.3, n.10, p.768-774, 2005.

QU, X. et al. Evaluation of anti-bacterial and wound

Rev. Bras. PI. Med., Campinas, v.17, n.4, supl. I, p.827-835, 2015. 
healing activity of the fruits of Amorpha fruticosa $L$. African Journal of Traditional, Complementary, and Alternative Medicines, v.10, n.3, p.458-468, 2013.

REBOLLAA. et al. Effect of Brassica oleracea in rats skin wound healing. Acta Cirurgica Brasileira, v.28, n.9, p.664-669, 2013.

REDDY, G.A.K. et al. Wound healing potential of Indian medicinal plants. Indian Journal of Pharmaceutical Sciences, v.2, p.75-87, 2012.

SANTOS, O.J.; TORRES, O.J.M. Phytotherapy evolution in the healing process in surgery. Arquivos Brasileiros de Cirurgia Digestiva, v.25, n.3, p.139, 2012.

SANTOS, J.S. et al. A Rosa Mosqueta no tratamento de feridas abertas: uma revisão. Revista Brasileira de Enfermagem, v.62, n.3, p.457-462, 2009.

SAUAIA FILHO, E.N. et al. Evaluation of the use of raw extract of Euphorbia tirucalli L. in the healing process of skin wounds in mice. Acta Cirurgica Brasileira, v.28, n.10, p. 716-720, 2013.

SEITÉ, S. et al. Importance of treatment of skin xerosis in diabetes. Journal of the European Academy of Dermatology and Venereology, v.25, n.5, p.607-609, 2011.

SHEEBA, M. et al. Wound healing activity of Cassia occidentalis L. in albino wistar rats. International Journal of Integrative Biology, v.8, n.1, p.1-6, 2009.

SOCIEDADE BRASILEIRADEANGIOLOGIAE CIRURGIA VASCULAR RJ (SBACVRJ). Número de homens com diabetes cresce em seis anos, 2013. Disponível em: http://www.sbacvrj.com.br/paciente/br/ler/612/85/ Not\%C3\%ADcias/cresce-numero-de-homens-comdiabetes. Acesso em: 18 set 2013.
SÜNTAR, I. et al. Appraisal on the wound healing and anti-inflammatory activities of the essential oils obtained from the cones and needles of pinus species by in vivo and in vitro experimental models. Jornal Ethnopharmacology, v.139, n.2, p.533-540, 2012.

THAKUR, R. et al. Practices in Wound Healing Studies of Plants. Evidence-Based Complementary and Alternative Medicine, v.2011, n.2011, p.1-17, 2011.

TRIVELLATO GRASSI, L. et al. From popular use to pharmacological validation: a study of the antiinflammatory, anti-nociceptive and healing effects of Chenopodium ambrosioides extract. Journal Ethnopharmacol, v.145, n.1, p.127-138, 2013.

VIEIRA, D.R.P. et al. Plantas e constituintes químicos empregados em Odontologia: revisão de estudos etnofarmacológicos e de avaliação da atividade antimicrobiana in vitro em patógenos orais. Revista Brasileira de Plantas Medicianais, v.16, n.1, p.135167, 2014.

VISWANATHAN, V. et al. A pilot study on the effects of a polyherbal formulation cream on diabetic foot ulcers. The Indian Journal of Medical Research, v.134, n.2, p.168-173, 2011.

WEBER, T.M. et al. Treatment of xerosis with a topical formulation containing glyceryl glucoside, natural moisturizing factors, and ceramide. Journal of Clinical e Aesthet Dermatology, v.5, n.8, p.29-39, 2012.

WHITE-CHU, E.F.; REDDY, M. Dry skin in the elderly: complexities of a common problem. Clinics in Dermatology, v.29, n.1, p.37-42, 2011. 